\title{
Development of Vesicle Pools during Maturation of Hippocampal Synapses
}

\author{
Marina G. Mozhayeva, Yildirim Sara, Xinran Liu, and Ege T. Kavalali \\ Center for Basic Neuroscience and Department of Physiology, University of Texas Southwestern Medical Center, Dallas, \\ Texas 75390-9111
}

\begin{abstract}
We studied the emergence of vesicle pool organization at developing hippocampal synapses by monitoring vesicle recycling and neurotransmitter release as well as examining electron micrographs. Our analysis suggests that presynaptic boutons go through three distinct functional states to mature. At the onset the synapses lack readily releasable vesicles although they possess a pool of recycling vesicles that can release neurotransmitters under strong stimulation. In the next stage the majority of these recycling vesicles switches to a functionally docked state and forms the readily releasable pool
\end{abstract}

(RRP). After assembly of the RRP, new vesicles build the reserve pool. At the mature state the size of the RRP increases linearly with increasing recycling pool size. Furthermore, this preferential filling of the RRP during early synapse maturation is reduced strikingly in synapses deficient in synapsin I and II. Taken together, these results expose a mechanism that ensures functionally effective allocation of a limited number of vesicles in a CNS synapse.

Key words: synapse maturation; readily releasable pool; synapsins; FM1-43; patch clamp; hippocampal culture
The clustering of synaptic vesicles around active zones is the initial structural sign of synapse formation. During synapse maturation the number of vesicles in these clusters increases in parallel with changes in the architectural complexity of synaptic boutons (Dyson and Jones, 1980; Amaral and Dent, 1981; Blue and Parnavelas, 1983; Vaughn, 1989). Although anatomical aspects of this process have been investigated, functional correlates of the increase in the number of synaptic vesicles are not clear. Several studies revealed that the number of vesicles in synapses and the distribution of synaptic release probabilities are nonuniform even among synapses formed by a single neuron, creating a diversity that is thought to be critical for central neuronal information processing (Hessler et al., 1993; Rosenmund et al., 1993; Harris and Sultan, 1995; Dobrunz and Stevens, 1997; Murthy et al., 1997; Schikorski and Stevens, 1997). This diversity may originate partly from the identity of postsynaptic targets (Maccaferri et al., 1998; Markram et al., 1998; Reyes et al., 1998), or it may be attributable to parent cell-dependent presynaptic factors and asynchronous development of synapse populations. Observations on developmental changes in short-term synaptic plasticity indicate synapse maturation as a critical factor that influences presynaptic function (Bolshakov and Siegelbaum, 1995; Pouzat and Hestrin, 1997; Hsia et al., 1998; Reyes and Sakmann, 1999). Presynaptic properties such as release probability; short-term facilitation, and depression are determined partly by the functional availability of vesicles for release. Limitations in the number of vesicles, especially during early synaptic development, may

Received Aug. 10, 2001; revised Oct. 3, 2001; accepted Nov. 6, 2001.

E.T.K is the Effie Marie Cain Endowed Scholar in Biomedical Research at the University of Texas Southwestern Medical Center. We thank Jason Pyle, Jürgen Klingauf, and Thomas Südhof for helpful discussions and suggestions during this study as well as Ilya Bezprozvanny, Ferenc Deak, and Susanne Schoch for critically reading this manuscript. Synapsin I and II double knock-out mice were generously provided by Dr. Thomas Südhof.

Correspondence should be addressed to Dr. Ege T. Kavalali, Center for Basic Neuroscience, University of Texas Southwestern Medical Center, 5323 Harry Hines Boulevard, Dallas, TX 75390-9111. E-mail: Ege.Kavalali@UTSouthwestern.edu. Copyright (C) 2002 Society for Neuroscience $0270-6474 / 02 / 220654-12 \$ 15.00 / 0$ exert physical constraints on synaptic responses and result in significant deviations from mature presynaptic physiology.

Studies in several secretory preparations and central synapses have classified vesicles with respect to their relative availabilities for release during stimulation (Neher and Zucker, 1993; Rosenmund and Stevens, 1996; Schneggenburger et al., 1999; Wu and Borst, 1999). The readily releasable pool of vesicles (RRP) is thought to be immediately available for release with the arrival of an action potential (AP); structurally, the RRP presumably includes vesicles that are juxtaposed directly to active zones. The reserve pool of vesicles that are anatomically distant from release sites can become functionally competent for release during prolonged stimulation and can replace vesicles in the RRP. The RRP and the reserve pool together make up the recycling pool of vesicles (RP), which corresponds to all vesicles capable of activitydependent recycling with stimulation. The number of vesicles contained in the RRP has been suggested as a critical parameter that regulates the probability of neurotransmitter release as well as several forms of short- and long-term plasticity (Zucker, 1989; von Gersdorff et al., 1997; Goda and Stevens, 1998; Wu and Betz, 1998).

In contrast to extensive studies on the maturation of postsynaptic properties in central synapses (Durand et al., 1996; Wu et al., 1996), far less is known about developmental modifications in the vesicular organization of presynaptic terminals. To establish a functional link between synaptic physiology and development of synaptic morphology, we systematically studied the emergence of vesicle pool hierarchy in a hippocampal culture preparation.

\section{MATERIALS AND METHODS}

Cell culture. CA3 $\rightarrow$ dentate gyrus regions were dissected from the hippocampi of 1- to 2-d-old Sprague Dawley rats or mice, and dissociated cultures were prepared according to previously published protocols (Kavalali et al., 1999b).

Electron microscopy. The cells were fixed for $30 \mathrm{~min}$ in $2 \%$ glutaraldehyde buffered with $0.1 \mathrm{M}$ sodium phosphate, $\mathrm{pH} 7.2$, at $4^{\circ} \mathrm{C}$. They were rinsed twice in buffer and then incubated in $1 \% \mathrm{OsO}_{4}$ for $30 \mathrm{~min}$ at room temperature. After being rinsed with distilled water, the specimens were 
stained en bloc with $2 \%$ aqueous uranyl acetate for 15 min, dehydrated in ethanol, and embedded in poly/bed812 for $24 \mathrm{hr}$. The $50 \mathrm{~nm}$ sections were poststained with uranyl acetate and lead citrate and were viewed with a JEOL 1200 EX transmission electron microscope.

Fluorescence imaging. Synaptic boutons were loaded with $400 \mu \mathrm{M}$ FM2-10 or $8 \mu \mathrm{M}$ FM1-43 (Molecular Probes, Eugene, OR; see experiments in Fig. 7C,D) under conditions described in Results. The modified Tyrode's solution that was used in all experiments contained (in $\mathrm{mM}$ ): $150 \mathrm{NaCl}, 4 \mathrm{KCl}, 2 \mathrm{MgCl}_{2}, 10$ glucose, 10 HEPES, and $2 \mathrm{CaCl}_{2}, \mathrm{pH} 7.4$ (310 mOsm). Hypertonic solution was prepared by the addition of 500 mM sucrose to the Tyrode's, and hyperkalemic solutions contained an equimolar substitution of $\mathrm{KCl}$ for $\mathrm{NaCl}$. Field stimulation was applied through parallel platinum electrodes immersed into the perfusion chamber, delivering $30 \mathrm{~mA}, 1 \mathrm{msec}$ pulses. All staining and washing protocols were performed with $10 \mu \mathrm{M}$ 6-cyano-7-nitroquinoxaline-2,3-dione (CNQX) and $50 \mu \mathrm{M}$ aminophosphonopentanoic acid (AP-5) to prevent recurrent activity. Images were taken after $15 \mathrm{~min}$ washes in dye-free solution in nominal $\mathrm{Ca}^{2+}$ to minimize spontaneous dye loss. In all experiments we selected isolated boutons $\left(\sim 1 \mu \mathrm{m}^{2}\right)$ for analysis and avoided apparent synaptic clusters (Kavalali et al., 1999b). Destaining of hippocampal terminals with hypertonic/hyperkalemic challenge was achieved by direct perfusion of solutions onto the field of interest by gravity (1-2 $\mathrm{ml} / \mathrm{min})$. Adjustment of hyperosmotic solution flow rate was critical to prevent alterations in fluid levels and fluorescence values during rapid solution exchanges. Fluorescence values were not distorted significantly by cell shrinkage during sucrose application. Images were obtained by a cooled-intensified digital CCD camera (Roper Scientific, Trenton, NJ) during illumination $(1 \mathrm{~Hz}, 15 \mathrm{msec})$ at $480 \pm 20 \mathrm{~nm}(505$ dichroic longpass, $535 \pm 25$ bandpass) via an optical switch (Sutter Instruments, Novato, CA). Images were acquired and analyzed with Metafluor Software (Universal Imaging, Downingtown, PA).

To determine the level of activity-independent fluorescence loss, we performed mock destaining experiments on FM2-10-labeled puncta in $5 \mathrm{~d}$ in vitro (DIV) cultures with consecutive applications of $4 \mathrm{~K}^{+}$with no added $\mathrm{Ca}^{2+}$ (as in Fig. $4 A ; n=2$ ). In the time course of a $500 \mathrm{sec}$ experiment we typically observed a $10 \%$ decrease in fluorescence. With the same fluorescence excitation and detection settings as in the experiments described in Figure $5 B$, this loss corresponded to $1.5 \pm 5.5 \mathrm{U}$. This decrease presumably includes factors such as photobleaching, continual washout of residual dye, and spontaneous release.

To estimate the number of vesicles corresponding to a particular fluorescence value, we stained sparsely membranous areas that contained thin axonal and dendritic branches. We measured the amount of $\Delta F$ in 50 distributed $1 \mu \mathrm{m}^{2}$ regions after a rapid washout of dye from these processes. The fluorescence emitted from a spherical synaptic vesicle of $40 \mathrm{~nm}$ in diameter was calculated with the assumption that $1 \mu \mathrm{m}^{2}$ regions corresponded to a cylindrical area of $1 \mu \mathrm{m}$ in diameter and length. $\Delta F$ measurements from distinct regions had similar distributions for given dye and image acquisition settings. These procedures were performed for both FM2-10 and FM1-43. Experiments were performed under constant fluorescence excitation and image acquisition settings. For dim puncta we acquired images by using $2 \times 2$ on-chip binning; these values were scaled back to normal settings (with a factor of 3.84) by the use of calibrations performed with fluorescent beads (Molecular Probes).

Electrophysiology. Synaptic responses were recorded from pyramidal cells by whole-cell configuration of the patch-clamp technique. Data were acquired with an Axopatch 200B amplifier and Clampex 8.0 software (Axon Instruments, Union City, CA). Recordings were filtered at $1 \mathrm{kHz}$ and sampled at $200 \mu \mathrm{sec}$. Pipette internal solution included (in $\mathrm{mM}$ ): 115 Cs- $\mathrm{MeSO}_{3}, 10 \mathrm{CsCl}, 5 \mathrm{NaCl}, 10 \mathrm{HEPES}, 0.6$ EGTA, $20 \mathrm{TEA}-\mathrm{Cl}, 4$ Mg-ATP, $0.3 \mathrm{Na}_{2} \mathrm{GTP}$, and 10 QX-314, pH 7.35 (300 mOsm). Picospritzer-delivered pulses of hypertonic sucrose $(+500 \mathrm{mOsm})$ were applied to proximal dendrites. For AP-dependent stimulation we used the same technique as in imaging experiments.

\section{RESULTS}

\section{Development of presynaptic terminals in a hippocampal culture system}

One of the most dramatic examples of synaptic growth in mammalian CNS occurs in synapses formed by mossy fiber projections of dentate granule cells (Amaral and Dent, 1981). Several anatomical properties of this projection that include robust growth of presynaptic terminals can be reconstituted in a dissociated cocul-
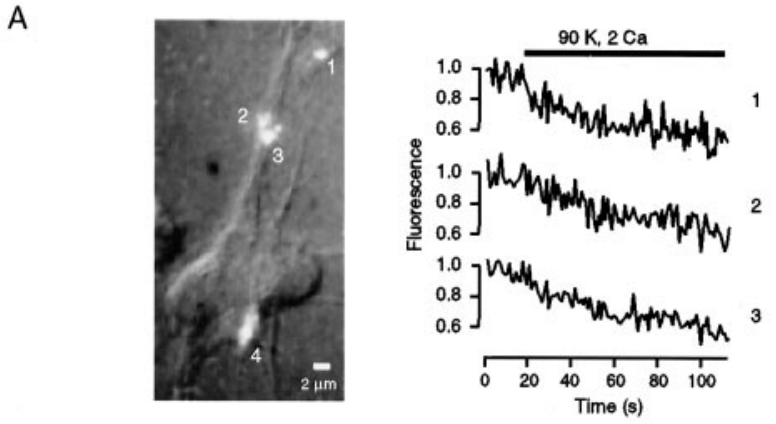

B

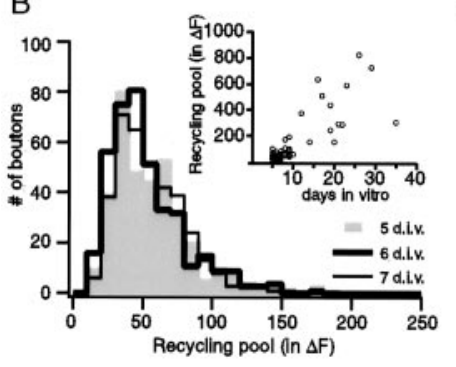

C

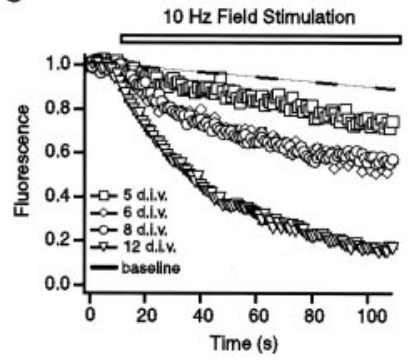

Figure 1. Characteristics of presynaptic maturation in a hippocampal culture system. $A$, Organization of synaptic puncta on proximal dendrites of a cluster of three neurons at 5 DIV. Superimposed differential interference contrast and fluorescence images have been acquired with $2 \times 2$ binning to increase the signal-to-noise ratio for these dim synapses. Background staining levels determined after four consecutive rounds of high $\mathrm{K}^{+}$application were subtracted from the fluorescence image. Destaining patterns of three FM2-10-labeled puncta are depicted on the right. Puncta with a diffuse pattern with no apparent center of mass were omitted from analysis (as in 4 ). $B$, Distributions of recycling pool sizes for individual boutons at 5,6, and 7 DIV do not show a significant difference. Inset, Plot depicts the increase in FM2-10 uptake during synaptic growth as a function of DIV. Each symbol represents an average recycling pool size (in $\Delta F$ ) of $\sim 60$ boutons from an experiment on a particular DIV. $C$, Average destaining patterns of synapses from 5 (85 boutons), 6 (78 boutons), 8 ( 83 boutons), and 12 DIV (105 boutons) stimulated with 10 $\mathrm{Hz}$ field stimulation. Error bars \pm SEM are within the symbols. The dashed line indicates the rate of fluorescence loss in control experiments in which boutons were subjected to multiple rounds of $4 \mathrm{~K}^{+}$solution in nominal extracellular $\mathrm{Ca}^{2+}$.

ture preparation from dentate gyrus and CA3 regions (Baranes et al., 1996; Kavalali et al., 1999b). To monitor the functional development of the vesicle population in these synapses, we used styryl dyes that label vesicles in an activity-dependent manner and provide excellent tools for the imaging of vesicle recycling at the level of individual synaptic boutons (Betz et al., 1996). In these cultures the formation of synapses and the clustering of synaptic vesicles could be detected with the use of uptake/release of styryl dyes starting at 5 DIV (Fig. $1 A$; also see Basarsky et al., 1994; Kavalali et al., 1999b). To quantify the growth of total recycling vesicle pool size, we loaded synaptic boutons with the fast-departitioning styryl dye FM2-10 $(400 \mu \mathrm{M})$ in the presence of a $45 \mathrm{mM} \mathrm{K}^{+} / 2 \mathrm{mM} \mathrm{Ca}^{2+}$ solution for $90 \mathrm{sec}$ to ensure maximal uptake of the dyes into individual synapses. The efficiency of this protocol in labeling the total recycling pool was verified by three successive applications that did not increase the amount of trapped fluorescence (data not shown). We determined the degree of loading after multiple applications of the $90 \mathrm{~mm} \mathrm{~K}^{+} / 2 \mathrm{~mm}$ $\mathrm{Ca}^{2+}$ solution to release the trapped fluorescence maximally and reach the lowest baseline levels. In these measurements the size of the recycling pool showed an increase nearly two orders of 
magnitude from 5 up to $35 \mathrm{DIV}$ (Fig. $1 B$, inset). The rise in RP size had a biphasic pattern without a significant increase between 5 and 7 DIV; this delay was followed by substantial growth in pool size from 8 DIV onward (Fig. $1 B$ ).

\section{The rate of styryl dye destaining increases during synapse maturation}

To obtain information on the kinetics of synaptic vesicle mobilization, we examined the destaining of individual synaptic boutons in response to AP firing at $10 \mathrm{~Hz}$ for $100 \mathrm{sec}$ induced by field stimulation, followed by multiple applications of high $\mathrm{K}^{+}$solution to determine background fluorescence (Fig. 1C). At 5 DIV the synapses showed a very slow rate of destaining with a time constant of $\sim 310 \mathrm{sec}$. Starting at $6 \mathrm{DIV}$, the average time constant decreased to $140 \mathrm{sec}$ and reached $130 \mathrm{sec}$ at 8 DIV. The distribution of destaining rates of individual boutons showed significant scatter at these stages. In the same culture, at 12 DIV, we measured a time constant of $\sim 50 \mathrm{sec}$, a value comparable with previous measurements in mature synapses in CA $1 \rightarrow \mathrm{CA} 3$ as well as dentate gyrus $\rightarrow$ CA3 cultures (Ryan and Smith, 1995; Kavalali et al., 1999b). Taken together, these observations indicate an increase in the availability of vesicles for release that parallels the maturation of individual synaptic boutons. To gain insight into this process, we used a strong stimulation with the bath application of a $90 \mathrm{mM} \mathrm{K}^{+} / 2 \mathrm{mM} \mathrm{Ca}^{+2}$ solution to distinguish kinetically the pools of vesicles with different availabilities for release. This depolarizing solution provides a massive influx of $\mathrm{Ca}^{2+}$ into the terminals by opening presynaptic $\mathrm{Ca}^{2+}$ channels independent of AP generation (Ryan et al., 1993) and results in a typical biphasic destaining profile (Klingauf et al., 1998). The initial phase of this destaining pattern is attributable to dye release from vesicles in a rapidly mobilizable pool in the order of seconds, which is typically larger than the RRP determined by hypertonic sucrose application (Pyle et al., 2000). The slow phase reflects physical replenishment of this rapidly releasable vesicle population from the reserve pool (Klingauf et al., 1998; Pyle et al., 2000). The developmental increase in the rate of destaining persisted under these conditions (Fig. 2A,B). This trend is illustrated clearly by the significant increase in the number of boutons with fast destaining kinetics from 5 to 7 DIV (Fig. $2 A$ ).

A sequential process by which, at the early stages of synapse maturation, vesicles in a slow recycling pool gradually form the RRP could explain this diversity in destaining kinetics. For instance, a slow monophasic destaining profile $(\tau=169 \mathrm{sec})$ of synapses at 5 DIV may indicate the presence of a slow recycling pool with few or no vesicles in the RRP. To analyze the synapses at 6 DIV, we used the multiple components of the cumulative histogram in Figure $2 A$ as a guide (open triangles) and grouped boutons into three categories. Of the synapses, $45 \%$ (184 of 404, denoted as $a$ in Fig. $2 A, B$ ) had a slow monophasic destaining similar to boutons at 5 DIV. Another $40 \%$ of the boutons ( $b$ in Fig. $2 A, B$ ) showed a biphasic destaining pattern. These synapses had, on average, $30 \%$ of their vesicles in a pool that could be mobilized with an average time constant of $11 \mathrm{sec}$. The remaining vesicles were in a slowly mobilizable pool similar to 5 DIV synapses ( $\sim 190 \mathrm{sec})$. Surprisingly, some boutons $(\sim 15 \%$, denoted as $c$ in Fig. $2 A, B)$ showed significantly fast destaining that could be fit with the values $\tau_{\text {fast }}=6.9$ and $\tau_{\text {slow }}=39.9 \mathrm{sec}$. The slow component of destaining in these synapses was faster than the slow component observed in mature synapses with a larger recycling pool size at $10 \mathrm{DIV}\left(\tau_{\text {fast }}=5.6\right.$ and $\left.\tau_{\text {slow }}=139.1 \mathrm{sec}\right)$. This
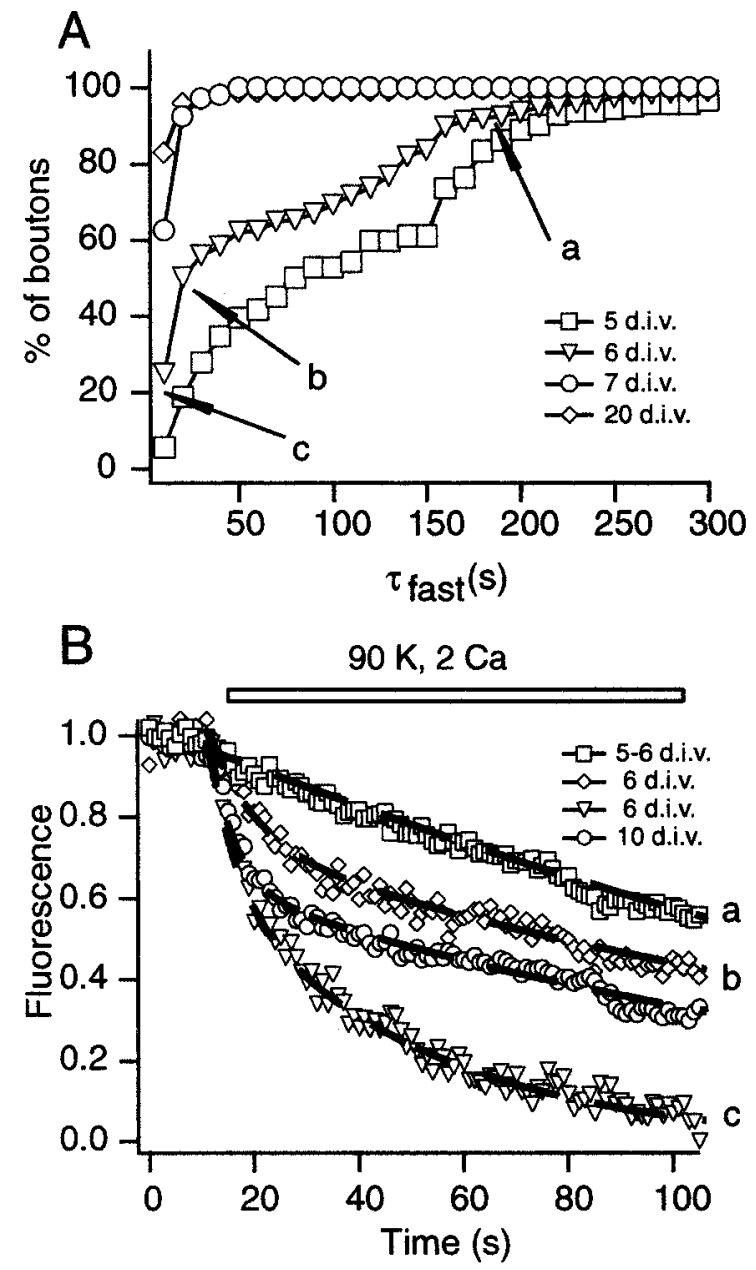

Figure 2. Analysis of FM2-10 destaining kinetics during synapse maturation. $A$, Cumulative histograms depicting the distribution of the fastest time constants that could be fit to destaining profiles induced by $90 \mathrm{~K}^{+}$stimulation at 5, 6, 7, and 20 DIV. $a, b$, and $c$ indicate the three distinct populations within the distribution at 6 DIV. Average destaining profile of each population is shown in $B$. $B$, Average normalized destaining patterns of synapses from 5,6 , and 10 DIV stimulated with $90 \mathrm{~K}^{+} / 2 \mathrm{Ca}^{2+}$. Boutons at $5 \mathrm{DIV}$ and some boutons at $6 \mathrm{DIV}$ had a slow destaining profile $(a)$. The remaining boutons at 6 DIV could be classified into two patterns (labeled as $b, c$ ) with the use of the histogram shown in $A$. The dashed lines represent fits to data with two exponential functions.

observation can be explained by the allocation of a substantial fraction of vesicles to the RRP $(\sim 80 \%)$. The deviation from the predicted fast and exhaustive destaining pattern may be attributable to the inactivation of voltage-gated $\mathrm{Ca}^{2+}$ channels that were induced during sustained depolarization. A decrease in $\mathrm{Ca}^{2+}$ influx was shown previously to limit the size of the fast component of destaining (Kavalali et al., 1999a).

In summary, this analysis points toward a process in which an early emerging slowly recycling pool of vesicles becomes docked gradually and forms the RRP. At this transient stage a large fraction of the vesicles in the synapse is in a functionally docked state and can be mobilized quite rapidly, as exemplified by the boutons grouped in $c$ (Fig. $2 A, B$ ). Increasing numbers of vesicles in the synapse result in the formation of a reserve pool. This final state underlies the mature kinetics of destaining (e.g., 10 DIV in Fig. 2B). 

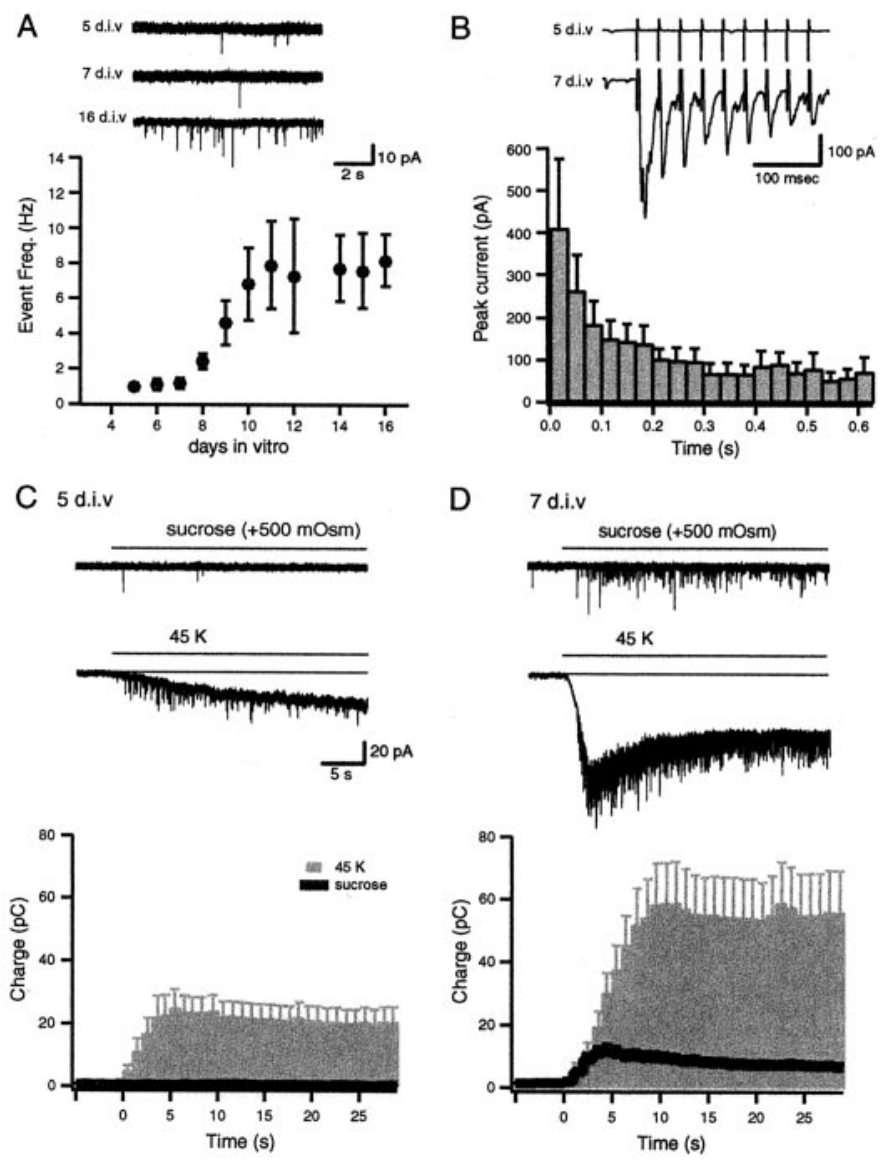

Figure 3. Delayed emergence of stimulation-dependent postsynaptic events during maturation. $A$, Representative recording traces depict spontaneous release events recorded at 5, 7, and 16 DIV. The graph depicts the increase in the frequency of spontaneous events between 5 and 16 DIV $(n=5-15$ for each symbol); note the sigmoidal rise in mini-frequency after 8 DIV. $B$, Traces from two representative experiments at 5 and 7 DIV. In contrast to 7 DIV, the cultures at 5 DIV were not responsive to AP stimulation. The graph depicts the decline in the amplitude of synaptic responses during $30 \mathrm{~Hz}$ stimulation at 7 DIV $(n=7)$. C, $D$, Comparison of whole-cell recording experiments from cells at 5 DIV $(C)$ and 7 DIV $(D)$. Hypertonic sucrose stimulation was not effective on cells that were tested at $5 \mathrm{DIV}$, whereas on the same cells $45 \mathrm{~K}^{+}$stimulation induced a substantial increase in event frequency at $5 \mathrm{DIV}$ and to a larger extent at 7 DIV. Baseline current drift induced under $45 \mathrm{~K}^{+}$perfusion was not sensitive to the subsequent application of blockers of glutamatergic and GABAergic transmission (data not shown) and was omitted from analysis. The bottom panels show cumulative charge transfer integrated over $1 \mathrm{sec}$ bins in response to hypertonic (area under the black line) and hyperkalemic ( gray bars) stimulation at $5 \operatorname{DIV}(n=7)$ and 7 DIV $(n=$ $5)$. Error bars indicate \pm SEM.

\section{Delayed emergence of synaptic responses to action potential and hypertonic stimulation}

If synapses at the earliest stages of maturation lack a functional set of readily releasable vesicles, as predicted by the above analysis, then they would be expected to be unresponsive to brief high-frequency AP stimulation short of mobilizing vesicles from the reserve pool. To test this prediction, we performed patchclamp recordings to detect neurotransmitter release from a population of synapses in response to $20 \mathrm{APs}$ at $30 \mathrm{~Hz}$. At $5 \mathrm{DIV}$ we could detect spontaneous release events at a frequency of $1 \mathrm{~Hz}$ $(0.88 \pm 0.57)$ on all of the cells that were tested $(n=15$; Fig. $3 A)$. However, synapses at this stage failed to respond to APs (Fig. $3 B$ ). At $7 \mathrm{DIV}$, in contrast to the absence of a significant increase in the frequency of spontaneous events, there were robust synaptic responses to $30 \mathrm{~Hz}$ stimulation with prominent depression in amplitude $(n=7$; Fig. $3 B$ ). Starting at $8 \mathrm{DIV}$, there was a significant increase in the frequency of spontaneous release that was correlated with the time course of synaptogenesis in this culture system (Kavalali et al., 1999b).

In parallel experiments we monitored the appearance of synaptic activity in response to an application of hyperosmotic solution $(+500 \mathrm{mOsm})$, an alternative means to mobilize vesicles in RRP. One of the critical advantages of this stimulation is its $\mathrm{Ca}^{2+}$ independence, which can help us discern alterations related to presynaptic machinery and vesicular organization from changes in $\mathrm{Ca}^{2+}$ influx through $\mathrm{Ca}^{2+}$ channels (Rosenmund and Stevens, 1996). At 5 and 6 DIV, pressure ejection of hypertonic sucrose solution onto synaptically dense areas on dendrites of pyramidal neurons, identified by FM2-10 staining, did not result in measurable synaptic responses in a substantial fraction of the cells that were tested (21 of 23; Fig. $3 C$ ). On cells in which we could not detect any activity in response to an application of hyperosmotic sucrose solution, we observed a substantial increase in the frequency of events caused by a $45 \mathrm{~K}^{+} / 2 \mathrm{Ca}^{2+}$ stimulation (Fig. $3 C$ ). Beginning at $7 \mathrm{DIV}$, we consistently could identify responses to hyperosmotic sucrose, which were present on every cell that was tested from 10 DIV onward (Fig. 3D). The appearance of sucrose responses after 7 DIV was associated with a marked increase in high $\mathrm{K}^{+}$-induced activity $(n=5$; Fig. $3 D)$. These results indicate that some synapses at 5 DIV lack a functional RRP although they possess a set of vesicles that can be released with strong stimulation.

\section{Estimation of the fraction of vesicles in RRP by sequential destaining of vesicle pools}

To extend these observations to the level of individual synaptic boutons, we performed fluorescence imaging experiments to determine the fraction of vesicles in the RRP within the recycling pool. After maximal loading with FM2-10 via high $\mathrm{K}^{+}$stimulation and a washout of surface dye, the presynaptic terminals were challenged sequentially to release the RRP and the reserve pool by hyperosmotic sucrose and high $\mathrm{K}^{+}$solutions, respectively (Fig. $4 A$ ). Previous studies in mature boutons have shown that the application of hyperosmotic solution to FM2-10-loaded boutons results in a fast destaining limited to $\sim 30 \%$ of the total pool, which corresponds to the size of the RRP (Pyle et al., 2000). Therefore, we estimated the fraction of the RRP by using the amplitude of the fast exponential decay in fluorescence induced by hyperosmotic shock. Here it should be noted that RRP size as estimated by hyperosmotic stimulation (30\%) is typically less than the size of the fast component of high $\mathrm{K}^{+}$-induced destaining (40-60\%), presumably because of fast replenishment and an increase in RRP size during $\mathrm{Ca}^{2+}$-dependent stimulation (Smith et al., 1998; Pyle et al., 2000). Among the population of synapses at 5 and 6 DIV that were identified positively by their ability to take up FM2-10, 43\% did not have discernible fluorescence drops to hyperosmotic sucrose application (Fig. $4 C, D$ ). The fraction of sucrose-unresponsive boutons decreased to $16 \%$ at $7 \mathrm{DIV}$, and virtually all synapses were sucrose-responsive by 9 DIV (Fig. 4D). Furthermore, in contrast to mature boutons these experiments also revealed a large scatter in ratios of sucrose responses to RP size from 5 up to 8 DIV. Some synapses could be destained almost completely with sucrose application, and they had minimal additional destaining with subsequent high $\mathrm{K}^{+}$applications (Fig. 4B). 

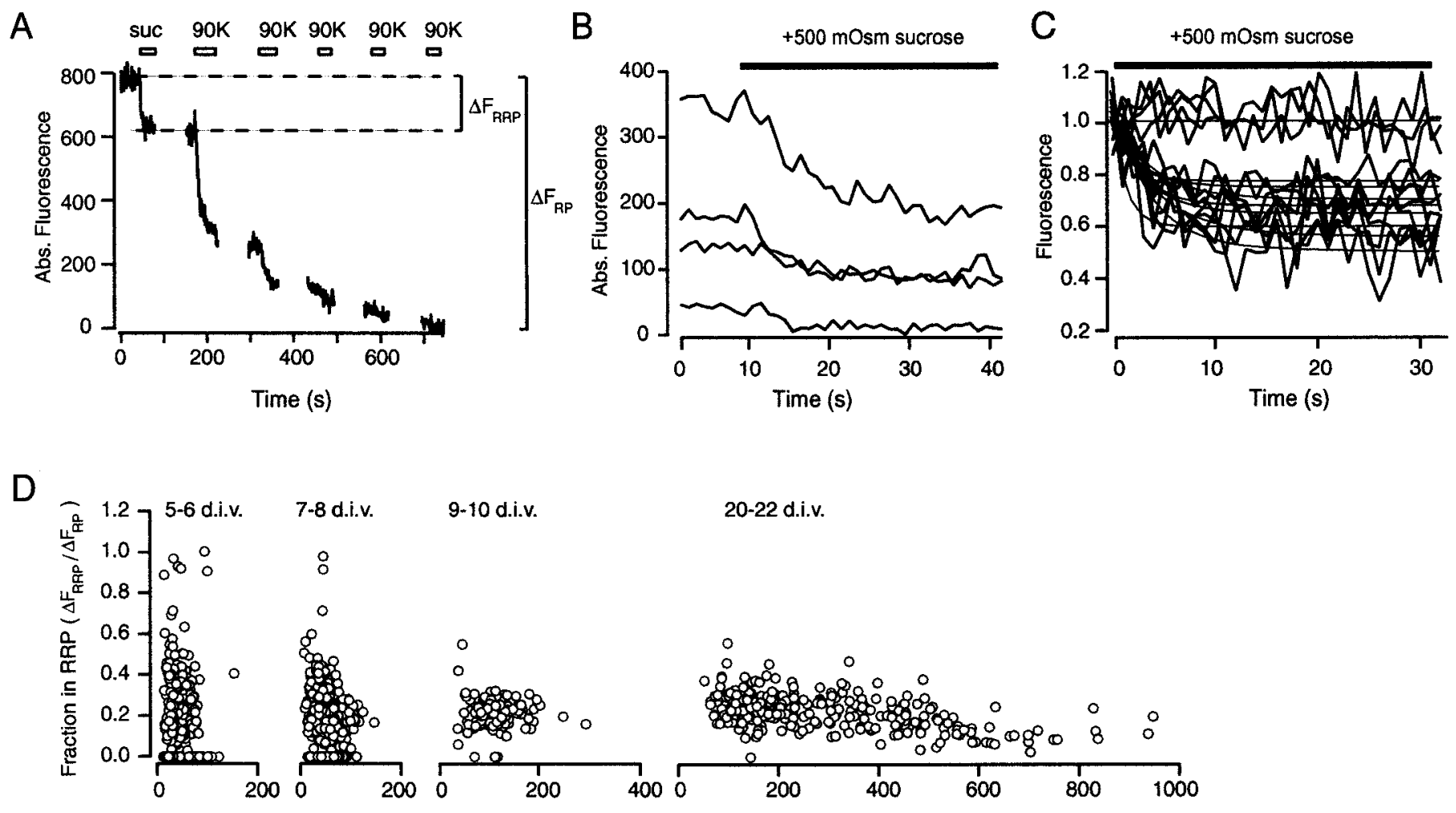

20-22 d.i.v.

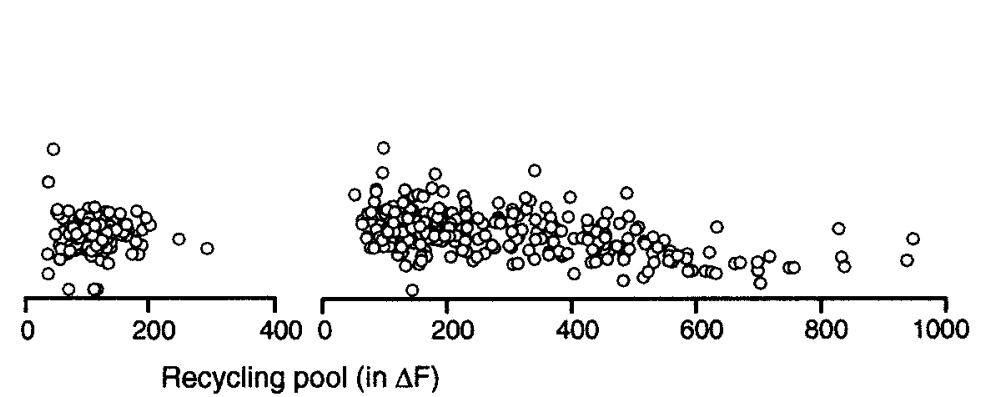

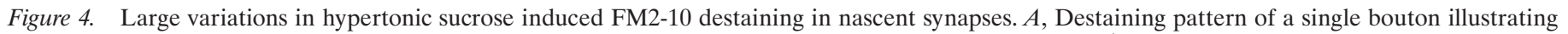

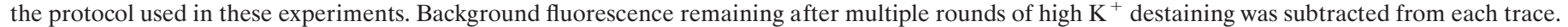

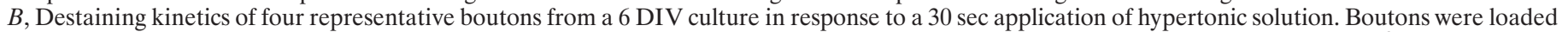

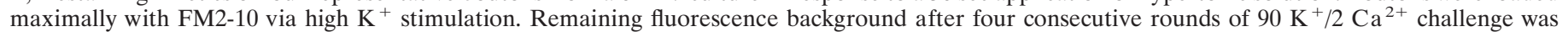

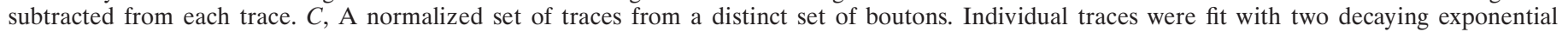

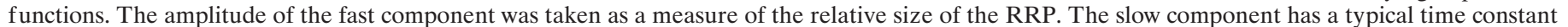

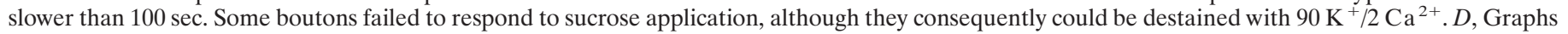

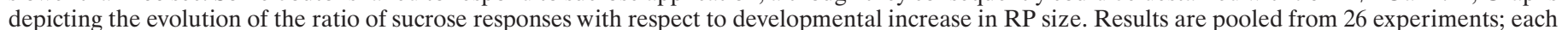

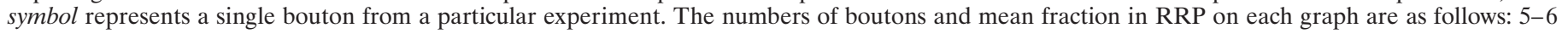
DIV, 453 boutons and 0.35; 7-8 DIV, 593 boutons and 0.23; 9-10 DIV, 129 boutons and 0.21; 20-22 DIV, 328 boutons and 0.22.

What is the origin of this scatter in the ratio of sucrose responses to total pool size at early stages of synapse maturation? As described previously, a dynamic organization process in which vesicles in a recycling but not functionally docked state gradually form a RRP would provide a potential explanation for this observation. To quantify the nature of this process with better accuracy, we need to consider the error introduced by fast endocytosis on FM dye-destaining kinetics (Klingauf et al., 1998; Kavalali et al., 1999a; Pyle et al., 2000). We calculated this error by using the $0.6 \mathrm{sec}$ membrane departitioning time constant for FM2-10 and the $1.1 \mathrm{sec}$ estimate for fast endocytosis at hippocampal synapses with exocytosis of RRP (Pyle et al., 2000). This calculation indicates that the sizes of the FM2-10 fluorescence drops we observed are $~ 80 \%$ of the actual size of the RRP. If we correct our measurements for this error, estimates for the size of the RRP for all of the synapses that have been analyzed would increase by $25 \%$. Especially at 5-8 DIV, a larger proportion of synapses will fall into the category in which $>50 \%$ of recycling vesicles are in the RRP. To verify these estimates, we quantified the amount of dye uptake, a measure less sensitive to fast endocytosis because of a millisecond time scale membrane partitioning rate of styryl dyes (Neves and Lagnado, 1999).

\section{Quantification of dye loading into RRP versus RP reveals distinct phases of vesicle organization}

In experiments conducted at 6 DIV we applied hypertonic stimulation $(+500 \mathrm{mOsm})$ in the presence of $400 \mu \mathrm{M}$ FM2-10 for 30 sec to load RRP and assessed the amount of loading by subsequent destaining, using multiple high $\mathrm{K}^{+}$stimulations. After a 5 min resting period, we labeled the same synapses with a $90 \mathrm{sec}$ application of $45 \mathrm{~K}^{+} / 2 \mathrm{Ca}^{2+}$ to load all recycling vesicles and determined the degree of loading in the same way (Fig. $5 A$ ). Figure $5 B$ shows the distribution of ratios of these two loading levels from 362 boutons. The data from each of the five experiments (Fig. 5C) that contributed to the cumulative plot in Figure $5 B$ show a clustered nature. This implies a local quasi-synchrony in synaptic development and supports a sequential process in the formation of vesicle pool hierarchy. To verify these results under experimental conditions with a higher signal-to-noise ratio, we repeated these experiments with FM1-43, a brighter analog of FM2-10, and obtained similar results (data not shown). Because of fast membrane partitioning both FM2-10 and FM1-43 can label the recycling pool equally, although the discrepancy between the two dyes emerges during destaining (Klingauf et al., 1998; Pyle et al., 2000).

To estimate the number of boutons that could not be labeled 

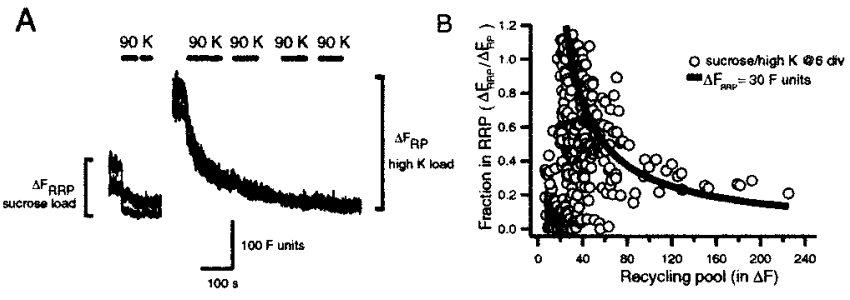

C

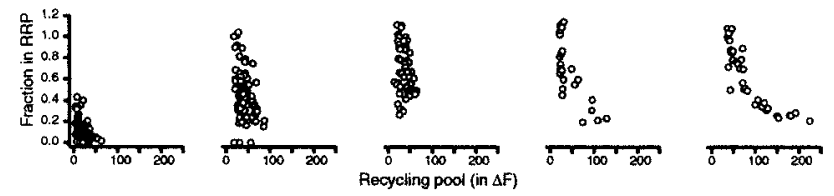

D

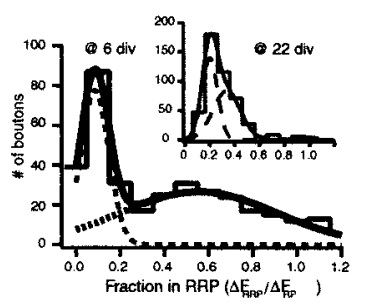

E

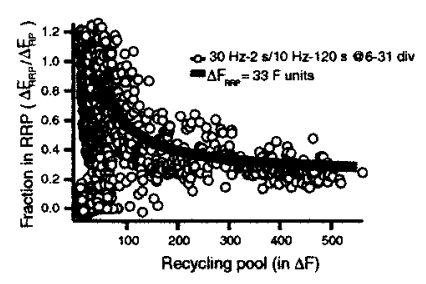

Figure 5. Fraction of vesicles in RRP declines with increasing recycling pool size. $A$, Sequential loading and destaining of three boutons illustrating the protocol that was used in these experiments. Background fluorescence was subtracted from each trace. $B$, Plot of the ratio of the size of the RRP to RP with respect to increasing size of the RP at 6 DIV $(n=5 ; 362$ boutons). RRP was stained with a $30 \mathrm{sec}$ application of $+500 \mathrm{mOsm}$ solution; the size of the RP was determined with $90 \mathrm{sec}$ high $\mathrm{K}^{+}$loading in the second round. The distribution of $R R P / R P$ values for $R P>40 \mathrm{U}$ could be fit with a hyperbolic function by minimizing the least square error $\left(\Delta F_{\mathrm{RRP}}=30 \mathrm{U}\right)$. According to our estimations this fluorescence value corresponds to nine vesicles. $C, \mathrm{RRP} / \mathrm{RP}$ distributions from five individual experiments that make up the plot in $B$. Note the clustered pattern of pool organization in each experiment. $D$, Distribution of RRP to RP ratios can be fit with two distinct Gaussian peaks. First is a sharp peak $\sim 0.08$, which significantly overlaps with baseline noise levels in these experiments; therefore, it presumably corresponds to sucroseunresponsive boutons. The second peak has a wide spread $\sim 0.60$. Inset, Nearly unimodal distribution of RRP to RP ratios from a set of mature boutons at 22 DIV. Gaussian peaks are centered on 0.19 and 0.33 , respectively. $E$, Loading RRP and RP with AP-dependent stimulations yielded similar results. The relative fraction of vesicles stained with FM2-10 by $30 \mathrm{~Hz}, 2$ sec stimulation was significantly larger for small boutons $\left(\Delta F_{\mathrm{RP}}<200 \mathrm{U}\right)$. The distribution of $\mathrm{RRP} / \mathrm{RP}$ values for $\mathrm{RP}>$ $40 \mathrm{U}$ was fit with a hyperbolic function, $\Delta F_{\mathrm{RRP}} / \Delta F_{\mathrm{RP}}=\left(33 / \Delta F_{\mathrm{RRP}}\right)+$ 0.22 , by minimizing least square error.

with hypertonic sucrose and thus did not possess a RRP, we first determined the background level of activity-independent fluorescence loss in a typical destaining experiment (see Materials and Methods). After hypertonic stimulation, loading levels of 169 boutons $(47 \%)$ were within $1 \mathrm{SD}$ of the mean background fluorescence loss $(1.5 \pm 5.5 \mathrm{U})$. These boutons subsequently could be identified by using maximal loading with high $\mathrm{K}^{+}$stimulation, and they had fluorescence values higher than 2 SDs above background (12.5 $\mathrm{U}$ for the dimmest bouton). In the remaining boutons, on average, a large fraction of the total recycling pool could be labeled with hypertonic stimulation (fraction in RRP, $0.60 \pm$ $0.25)$. In these boutons the fluorescence trapped during sucrose loading was at least 1.5 SD above background; thus these $\mathrm{RRP} / \mathrm{RP}$ values were distorted minimally by baseline noise. The divergence between the two sets of boutons can be visualized clearly in the histogram built for RRP/RP ratios (Fig. $5 D$ ). This distribution contained two widely separated populations. The sharp peak around the ratio of 0.08 corresponded to boutons classified as nonsucrose-responsive, whereas the broad peak centered at the ratio of 0.6 represented the remaining sucroseresponsive synapses. In contrast, a histogram built for RRP/RP ratios for boutons at 22 DIV showed a nearly unimodal distribution that could be fit with two closely spaced peaks at 0.19 and 0.33 , respectively (Fig. 5D, inset).

In parallel with increases in levels of fluorescence trapped within the RP, the number of vesicles in the RRP remained relatively constant, and RRP/RP steadily declined to $0.3(29.7 \pm$ $0.1 \%$, for boutons $>80 \mathrm{U})$. The distribution of RRP/RP values for boutons with RP $>40 \mathrm{U}$ could be fit loosely with a hyperbolic function in which the amount of RRP fluorescence was constant at $30 \mathrm{U}\left(r^{2}=0.20\right)$. The number of vesicles corresponding to this fluorescence value was estimated to be $\sim 9$ (see Materials and Methods). The decline in RRP/RP ratio agrees with the predictions of the model described above in which, after the formation of the RRP, the number of vesicles in this pool remains constant, and new vesicles build a reserve pool.

To verify the validity of these observations under physiological synaptic stimulation patterns, we used field stimulation-induced APs to stain vesicles. The design of these experiments was the same as described in Figure $5 A$. Instead of hypertonic stimulation, we labeled the RRP with $60 \mathrm{APs}$ at $30 \mathrm{~Hz}$, and the total RP was labeled with $1200 \mathrm{APs}$ at $10 \mathrm{~Hz}$. Figure $5 E$ depicts pooled results from experiments conducted at 6 DIV $(n=3)$ and 31 DIV $(n=$ 3). A combination of experiments from these two stages of maturation encompasses a wide range of RP sizes and illustrates the trend in the decline of RRP/RP with synaptic growth. When we applied the criteria described above, among the 471 boutons that were analyzed 123 (26\%) were unresponsive to $30 \mathrm{~Hz}, 2 \mathrm{sec}$ stimulation, indicating an absence of a functional RRP. Remaining boutons within the same range of RP values had an average $\mathrm{RRP} / \mathrm{RP}$ between 0.7 and 0.8 , slightly higher than results that were obtained from experiments that used hypertonicity and high $\mathrm{K}^{+}$-induced loading.

At later stages of maturation, because of an increase in the number of reserve vesicles (RP-RRP), the RRP to RP ratio converged to $\sim 0.3$ in agreement with previous studies (Murthy and Stevens, 1999; Pyle et al., 2000). Similar to in Figure 5B, the distribution of RRP/RP values (for RP $>40 \mathrm{U}$ ) could be described reliably with a hyperbolic function in which RRP size is constant $\left(\Delta F_{\mathrm{RRP}}=33 \mathrm{U}, \sim 9\right.$ vesicles; $\left.r^{2}=0.98\right)$. However, to account for preservation of the $\mathrm{RRP} / \mathrm{RP}$ ratio at $\sim 0.3$ for synapses with large RPs ( $>200 \mathrm{U})$, we modified the equation that we used to fit the distribution at 6 DIV in Figure $5 B$ with the addition of a horizontal asymptote at $\mathrm{RRP} / \mathrm{RP}=0.22$. This analysis indicates a phase of synchronous increase for RRP size and RP size in mature synapses with a large population of vesicles.

Interpretation of experiments that quantify an absolute amount of loading for a given vesicle pool in consecutive rounds of staining relies on two assumptions. The first assumption necessitates maximal loading of both types of vesicle pools with minimal dye loss during the washout period; in addition, these experiments require that the RP size remain stable for two rounds of staining/destaining. To verify the validity of these assumptions, we loaded synapses to saturating levels in two consecutive rounds. The ratio of first loading to second loading for synapses across different sizes and levels of maturation was distributed between 0.9 and 1.1 (data not shown; 548 boutons; $n=6$ ).

Previous studies have shown that vesicles in the RRP and the 
A

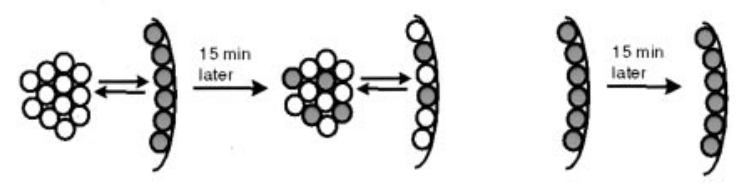

B

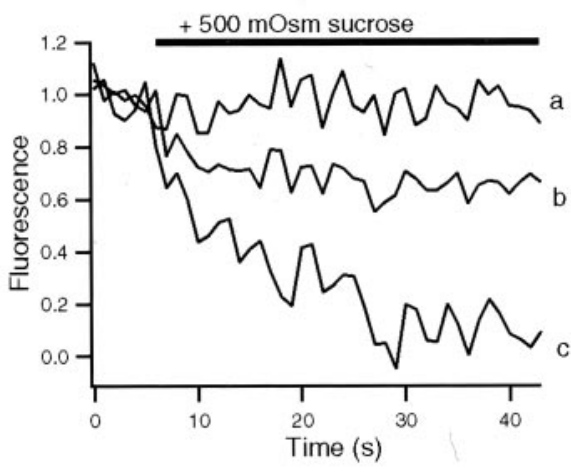

C

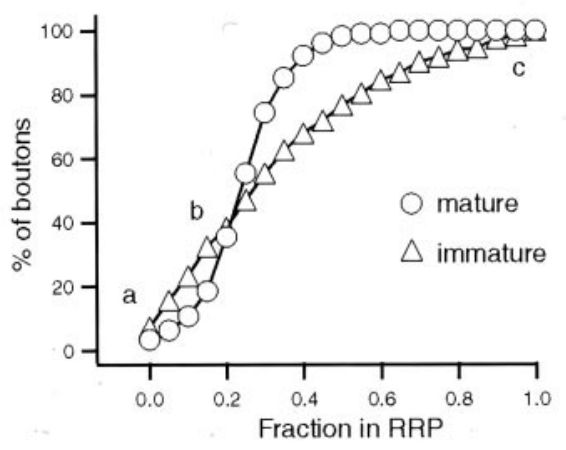

Figure 6. Mixing of RRP vesicles with the RP reveals diversity of vesicle pool organization. $A$, Diagram depicting the rationale behind the experiments. We used the dilution of stained RRP vesicles among all recycling pool vesicles to estimate the ratio of the two pools. This method does not rely on maximal loading of synapses. $B$, Representative destainings of immature synapses from a 6 DIV culture in response to hypertonic solution. Each trace is an example of the indicated locations on the distribution that is shown in $C$. After sucrose application the boutons were destained to their baseline values with $90 \mathrm{~K}^{+} / 2 \mathrm{Ca}^{2+}$. $C$, Cumulative histograms comparing the distribution of relative ratios of sucrosereleasable vesicles after sucrose-induced loading between mature $(n=3$; 15-20 DIV) and immature synapses ( $n=3$; 6 DIV).

reserve pool are in a dynamic equilibrium; therefore, in the steady state the vesicles that belong to the RRP at a given time point will be diluted among all vesicles in the synapse. This result allows for the use of nonmaximal loading conditions to uncover the relative distribution of vesicles between RRP and reserve pools. For this approach we stained the RRP vesicles with hypertonic sucrose application, followed by a 15 min washout period. This waiting period provided extensive time for stained vesicles that once belonged to the RRP to be mixed equally within the recycling pool (Ryan and Smith, 1995; Murthy and Stevens, 1999; Pyle et al., 2000). Under these conditions an application of hypertonic sucrose solution should result in a fluorescence drop in the same proportion to the ratio of vesicles between the RRP and RP (Fig. $6 A$ ). In the case of the mature synapses a second hypertonic challenge destained $\sim 25 \%$ of the previously stained RRP, indicating that $75 \%$ of the vesicles in the initial RRP have joined the reserve pool in agreement with previous destaining experiments (15-20 DIV; 231 boutons; Fig. 6C). Experiments in immature synapses (6 DIV; 150 boutons) showed a wider distribution, with significant deviation from mature boutons around a ratio of 0.5 (Fig. 6C; $p<0.005$, Kolmogorov-Smirnov test). As expected, the number of boutons that could not be destained with sucrose decreased significantly to $5 \%$, because this experimental paradigm selects for boutons that can be loaded only with sucrose (Fig. 6C). The presence of a few boutons with sucrose failures may indicate a level of instability in the organization of the RRP at these early stages.

\section{The role of synapsins in allocation of synaptic vesicles into RRP}

Our experiments so far support a scenario in which the recruitment of recycling vesicles to available functional docking sites at early stages of maturation results in assembly of the RRP. Potential molecular mediators of this process could be synaptic vesicleassociated phosphoprotein synapsins (Fernandez-Chacon and Südhof, 1999). These proteins are postulated to regulate vesicle recruitment to the active zone during repetitive stimulation. They also regulate the number of synaptic vesicles in central synapses (Rosahl et al., 1993, 1995; Pieribone et al., 1995; Ryan et al., 1996) (for review, see Hilfiker et al., 1999).

To investigate whether synapsins are involved in steady-state recruitment of vesicles to the RRP, we performed experiments on cultures obtained from synapsin I and II knock-out mice. We quantified the ratio of RRP to RP at individual synapses from 5 to 7 DIV cultures, using hypertonic sucrose and high $\mathrm{K}^{+}$dependent stimulation. Both sequential destaining and doubleloading experiments gave similar results. Synapses in cultures from age-matched mice had a distribution of RRP/RP ratios comparable with results from rat cultures (Fig. 7A). In contrast, experiments performed in cultures obtained from synapsin I and II double knock-out mice (Fig. $7 B, D$ ) had a significant reduction in the fraction of vesicles in RRP. This reduction was significantly pronounced in synapses with small RP sizes $\left(\Delta F_{\mathrm{RP}}<200 \mathrm{U}\right.$; Fig. $7 B$, inset; $p<0.001$ Kolmogorov-Smirnov test), indicating a deficiency in the recruitment of vesicles to functional docking sites. These results suggest that the large RRP/RP ratios we observed for small synapses are attributable to a synapsindependent process that recruits vesicles to the RRP.

To test whether the reduction in RRP size in immature knockout synapses also holds for small synapses at late developmental stages, we used FM1-43, a brighter analog of FM2-10. FM1-43 enabled more effective detection of boutons with small pool sizes in double-loading experiments (as in Fig. 5A). At 22 DIV, synapsin I- and II-deficient synapses showed a linear growth of RRP size with respect to increasing size of the RP (Fig. 7D) (RRP/ $\mathrm{RP}=0.14 \pm 0.07 ; r^{2}=0.81 ; 210$ boutons; $\left.n=4\right)$. The increase in the size of the recycling pool in synapsin knock-outs was relatively modest compared with wild-type synapses. This result in part may be attributable to a declustering of reserve pool vesicles as observed in other experimental settings in which synapsin I function was impaired ( $\mathrm{Li}$ et al., 1995; Pieribone et al., 1995). In contrast, distribution of RRP to RP ratios in wild-type synapses at 22 DIV displayed a hyperbolic decline with respect to increasing RP sizes because of enhanced detection of small boutons with FM1-43 (Fig. $7 C)\left(\Delta F_{\mathrm{RRP}}=88 \mathrm{U}, \sim 10\right.$ vesicles for FM1-43; $r^{2}=0.86 ; 466$ boutons; $\left.n=4\right)$. Among 466 synapses that we analyzed, 52 of them had RRP to RP ratios above $0.4(11 \%$; Fig. $7 D$, inset; $p<0.001$, Kolmogorov-Smirnov test). These results indicate that the deficit in the population of the RRP with recycling vesicles is still detectable at mature stages in synapses 

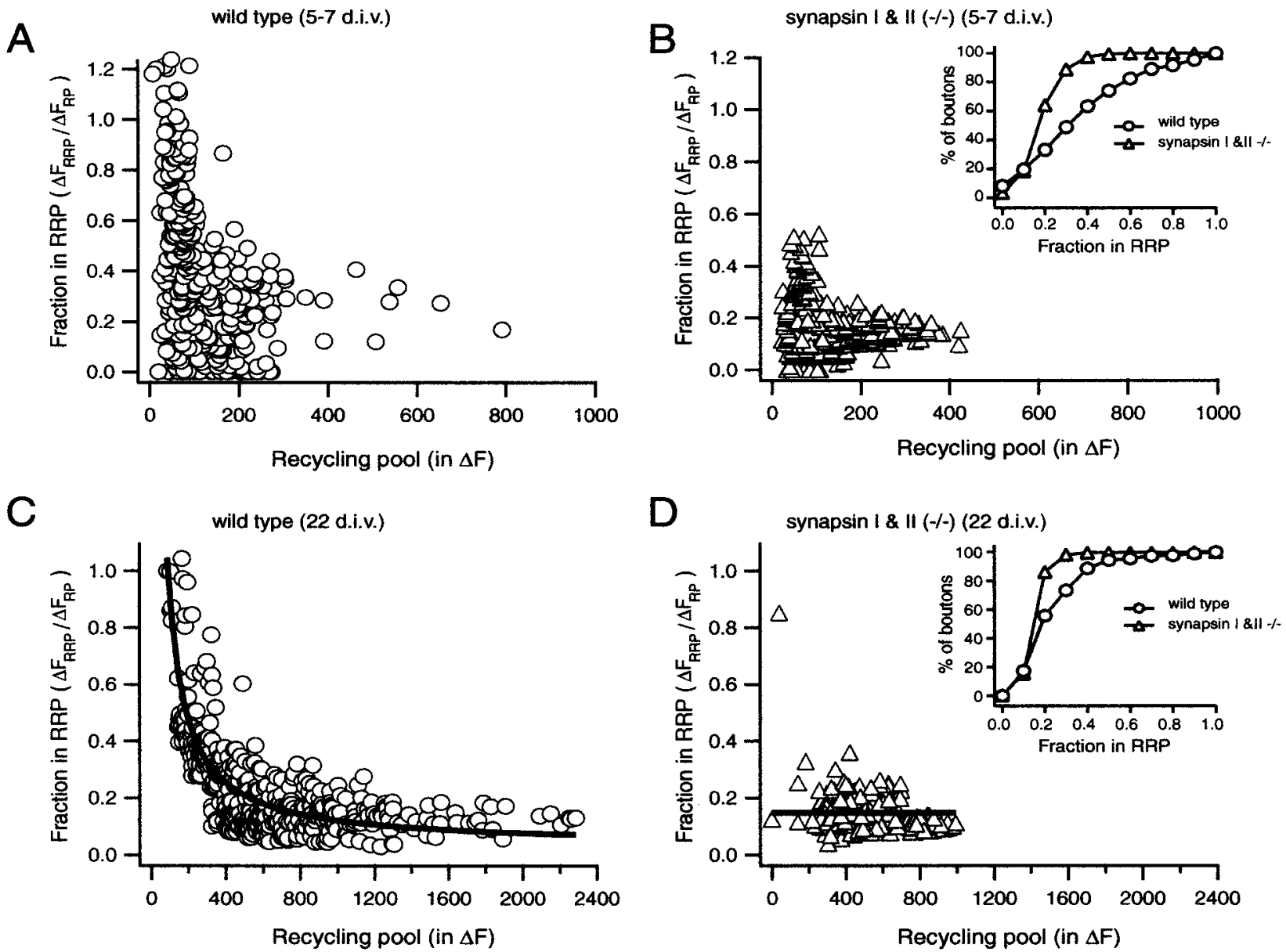

Figure 7. Synapsin I and II knock-outs are deficient in the population of RRP. $A$, Combined results from sequential destaining (as in Fig. $4 A$ ) and double-staining experiments (as in Fig. 5A) conducted on 5-7 DIV cultures obtained from mice (395 boutons). Both types of experiments were conducted by using hypertonic solution and high $\mathrm{K}^{+}$stimulation as described previously. $B$, Similar experiments performed on synapsin I- and II-deficient synapses (749 boutons) showed a significant reduction in the fraction of vesicles in RRP [Fraction in RRP; $0.20 \pm 0.08$ (mutant) vs $0.45 \pm 0.27$ (wild type)]. Mean RP size was not significantly different between mutant and wild-type synapses at this stage [108 (mutant) vs 119 (wild type) $F$ units[. Inset, Cumulative histograms comparing fraction of vesicles in RRP in wild-type and mutant synapses $(p<0.001$, Kolmogorov-Smirnov test). $C$, Distribution of the fraction of vesicles in RRP in wild-type synapses sequentially loaded with FM1-43 (protocol as in Fig. $5 A$ ). Note the presence of a small number of boutons $(11 \%)$ with large RRP to RP ratios in a mature population at 22 DIV. The continuous line represents a hyperbolic fit to the distribution of control synapses in which an absolute amount of loading with sucrose was constant at 88 fluorescence units ( $\sim 10$ vesicles). D, Boutons from synapsin I and II knock-outs had a restricted distribution of RRP/RP ratios $\sim 0.14$ (horizontal line), with a smaller overall RP size as determined by FM1-43 [mean RP size; $501 F$ units (mutant) vs $646 F$ units (wild type)]. Inset, Cumulative histograms comparing results from wild-type and mutant synapses $(p<0.001$, Kolmogorov-Smirnov test).

with small recycling pools. At these mature stages, in both wildtype and synapsin-deficient synapse populations, we could not detect any sucrose-unresponsive boutons despite our consistent detection of such boutons at 6 and 7 DIV.

\section{Morphological correlates of vesicle pool organization during synaptic development}

To investigate the parallels in ultrastructural organization of synaptic vesicles in developing presynaptic terminals and functional measurements, we examined electron micrograph sections (EMs) obtained from developing hippocampal cultures. At 5 DIV, $74 \%$ of vesicle clusters detectable in EMs were not associated clearly with the surface membrane or active zones (Fig. $8 A, B)$. These clusters may account for the slowly recycling pool we detected at this early stage of development. The rest of the EM sections had docked vesicles and were morphologically similar to the majority of synapses at 6 DIV. In EMs that were obtained at $6 \mathrm{DIV}$, the percentage of these nondocked vesicle clusters decreased to $40 \%$ (Fig. $8 C, D$ ). In this set of sections we also could detect a significant number of vesicle clusters in which most of the vesicles were within $100 \mathrm{~nm}$ of the active zone (Fig. $8 C$, middle). Among synapses with more mature appearance, we have observed cases in which there is a clear gap $(\sim 200 \mathrm{~nm})$ between a vesicle cluster near membrane surface and a distant "reserve" vesicle pool (Fig. 8C, right). Analysis of EMs at 20 DIV showed a large increase in the total number of vesicles per section (at $20 \mathrm{DIV}, 52.7 \pm 28.5$ vesicles, $n=124$ boutons; at $6 \mathrm{DIV}$, $27.2 \pm 22.7$ vesicles, $n=134$ boutons), and the percentage of vesicle clusters without morphologically docked vesicles decreased to $12 \%$.

When we quantified the ratio of the number of morphologically docked vesicles to the total number of vesicles detectable per section, we observed distributions analogous to the results of 
A

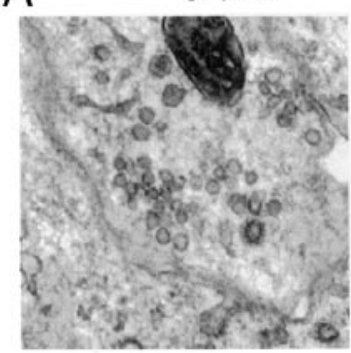

C

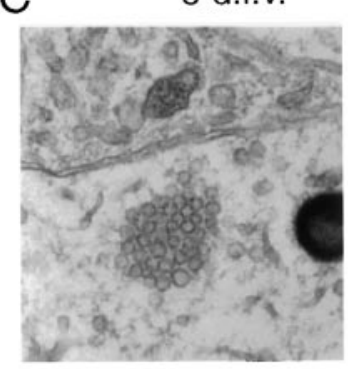

E

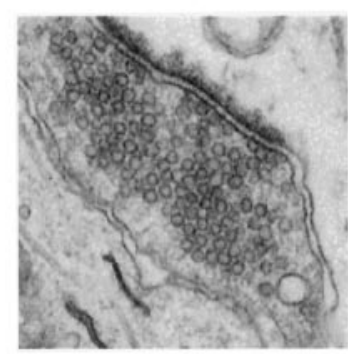

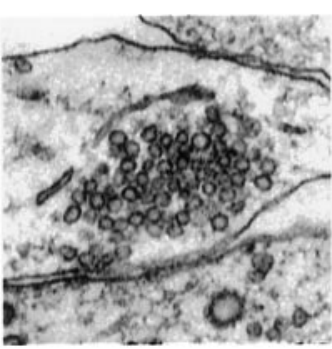

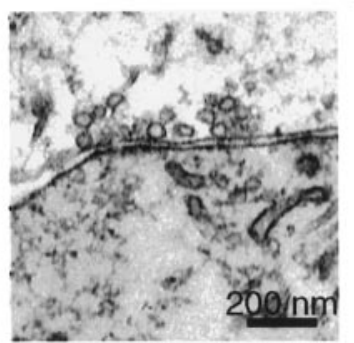

B
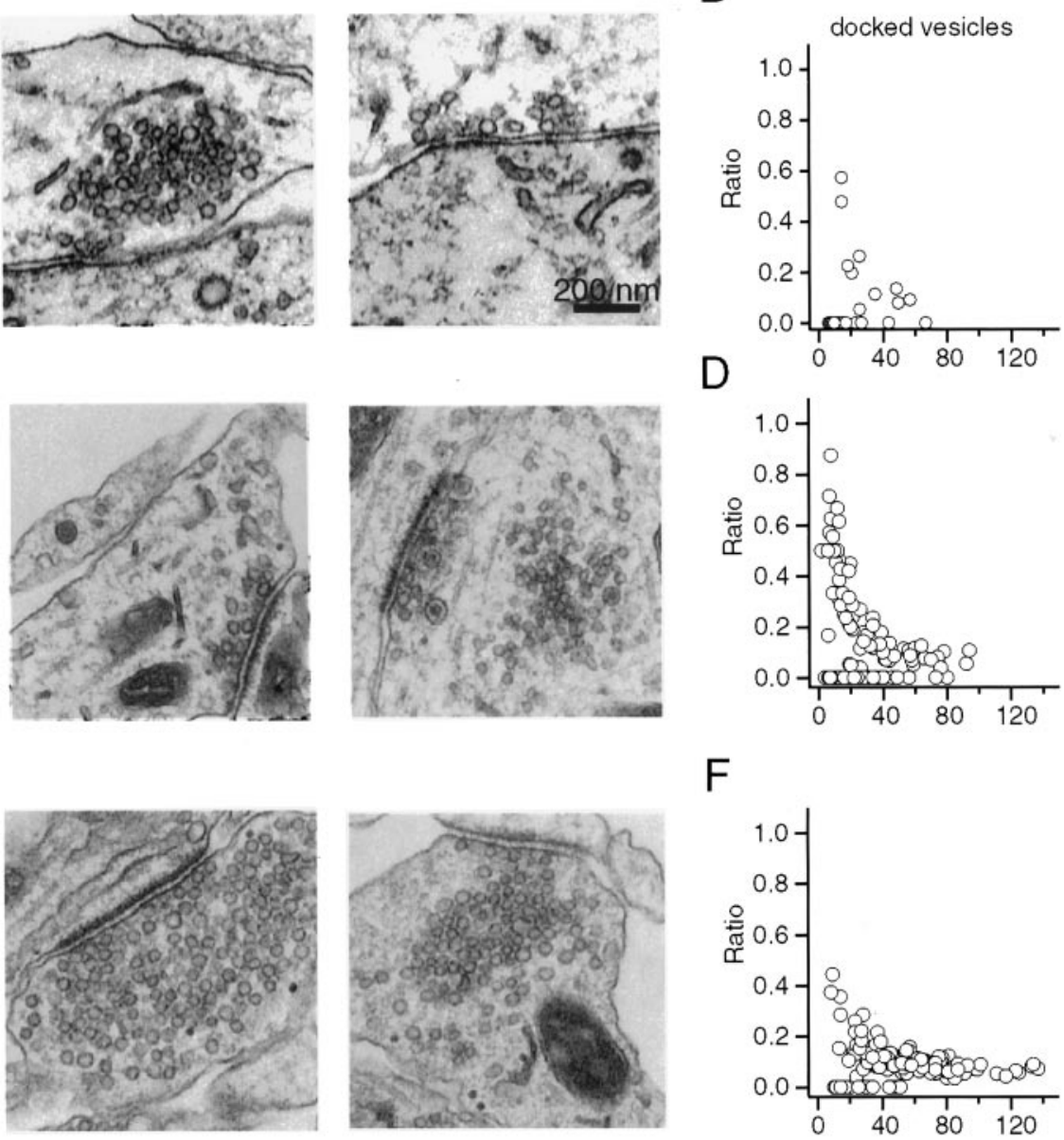

F

vesicles within 100nm of active zone

$\Delta$

$\Delta$

$\Delta \Delta \Delta$

$\Delta \Delta^{\Delta}$
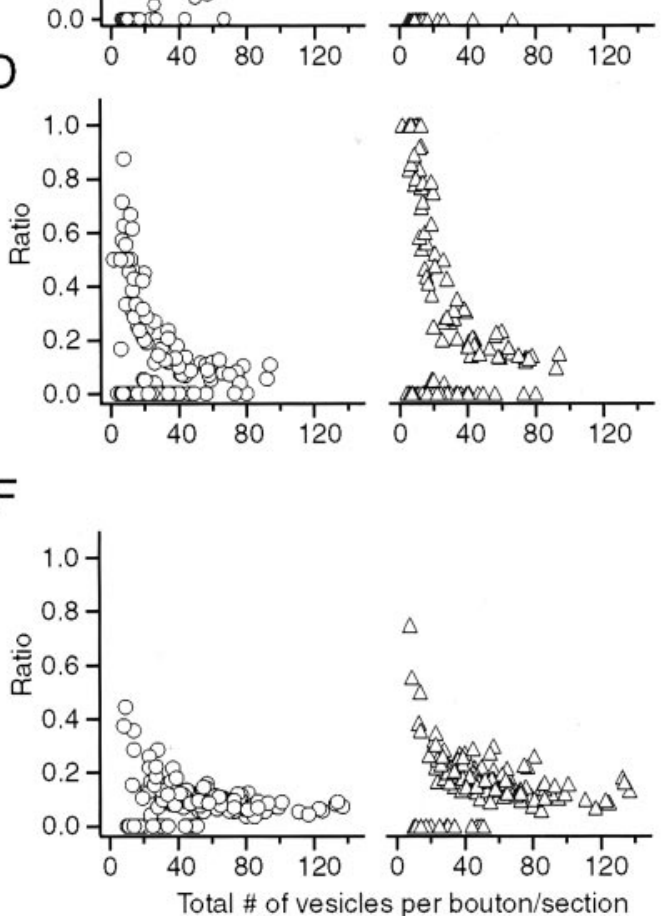

Figure 8. Ultrastructural analysis of vesicle organization reveals parallels with functional measurements. $A, B$, Electron micrographs depicting distinct morphological organization of vesicle clusters at 5 DIV. Of the synaptic vesicle clusters detected at this stage, $74 \%$ did not contain docked vesicles (total number of vesicles in a cluster, $14.7 \pm 12.5 ; n=51)$, whereas the rest of the synapses had docked vesicles $(3.8 \pm 1.4 ; n=13)$. $C, D$, At 6 DIV $40 \%$ of the vesicle clusters were not associated clearly with active zones as in 5 DIV (left $)$. In some synapses most of the vesicles were within $100 \mathrm{~nm}$ of the active zone (middle). Among synapses with a more mature appearance there was a clear gap $(\sim 200 \mathrm{~nm})$ between vesicle cluster near the membrane surface and a distant reserve vesicle pool (right). D, Left, The ratio of the number of docked vesicles plotted against the total number of vesicles (mean ratio, $0.30 \pm 0.20) . D$, Right, The ratio of vesicles within $100 \mathrm{~nm}$ of the active zone to the total in the same boutons (mean ratio, $0.50 \pm 0.33)$. The average values exclude sections without morphologically docked vesicles. $E$, $F$, Analysis of synapses in a 20 DIV culture preparation showed an increase in the total number of vesicles per section. Only $12 \%$ of vesicle clusters had no distinguishable docked vesicles, and the ratio of the number of docked vesicles versus the total number of vesicles decreased to $0.11 \pm 0.07$ (for vesicles within $100 \mathrm{~nm}, 0.19 \pm 0.10$ ).

fluorescence imaging experiments from immature and mature synapse populations (Fig. $8 B, D, F)$. At 6 DIV in sections with detectable docked vesicles, on average, $50 \%$ of vesicles were within $100 \mathrm{~nm}$ of the active zone $(0.50 \pm 0.33)$; this ratio was $19 \%$ for mature synapses $(0.19 \pm 0.10$; compare graphs on the right in Fig. $8 D, F)$. We also performed the same analysis on EM sections obtained from synapses at 6 DIV that lack synapsin I and II. Morphologically, these synapses were similar to their 6 DIV control counterparts in terms of the ratio of docked versus total number of vesicles (mean ratio, $0.60 \pm$ 0.24 ), although there was an approximately twofold reduction in the total number of vesicles $(15.2 \pm 9.3$ vesicles; $n=60$ sections). The absence of significant differences in the ratio of morphologically docked vesicles to the total vesicle pool, as projected from functional observations, restricts the role of synapsins to functional maturation of vesicles at the active zone during development.

\section{DISCUSSION}

\section{Distribution of synaptic vesicles into distinct pools during synapse maturation}

In this study by means of optical, electrophysiological, and ultrastructural analysis, we monitored the emergence of the readily releasable pool after synaptogenesis. In optical recordings the RRP was probed by fast kinetics of high $\mathrm{K}^{+}$-induced styryl dye destaining in addition to destaining and staining induced by hyperosmotic or brief AP stimulation. Electrophysiological experiments used the same stimulation protocols. Taken together, the results from these multiple experimental approaches suggest a developmental scheme in which presynaptic boutons visit three distinct states to attain their mature organization (Fig. 9). The serial progression of this scheme is supported by the strong correlations between in vitro age and recycling pool size and a particular pool organization in addition to quasi-synchronous 
Figure 9. A sequential model for emergence of vesicle pool organization during synaptic development. state 1, Depicted are synapses with recycling vesicle clusters before formation of the RRP. state 2, Shown is a transient state in which most of the functional docking sites within the active zone are populated by vesicles. The transition to state 2 may have stable intermediates, as indicated by the small arrows. To reach state 3, the number of vesicles in the synapse increases to build a reserve pool. In later stages of development the size of $\mathrm{RRP}$ and RP increases in parallel.

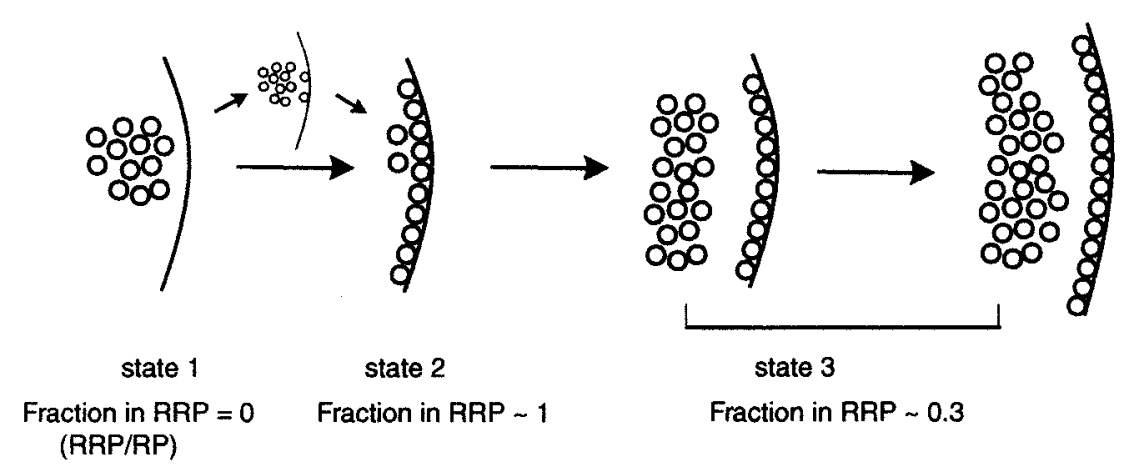

detection of a particular phase of pool organization in individual experiments (Fig. 5C). This local synchrony may be attributable to the factors such as maturity of parent neurons, the timing of initial synaptic contacts, and extent of process outgrowth in a particular region.

First state defines an early stage in maturation that follows closely the initial clustering of vesicles during synaptogenesis. Synapses in this state have a set of vesicles capable of dye uptake and release in response to high $\mathrm{K}^{+}$or long trains of APs. However, they do not possess a set of functionally docked vesicles that can be detected by hypertonic sucrose or $30 \mathrm{~Hz}, 2 \mathrm{sec}$ AP stimulation. This finding also is supported by the fact that perfusion of hypertonic sucrose solution onto synapses at 5-6 DIV did not result in detectable electrophysiological responses. Ineffectiveness of hypertonic stimulation at this stage supports the reorganization of vesicle pools, although we cannot exclude potential parallel changes in localization of presynaptic $\mathrm{Ca}^{2+}$ channels or the extent of $\mathrm{Ca}^{2+}$ influx. Lack of the RRP results in a very low probability of release and renders these boutons silent under low-frequency or short-duration stimulations.

Our analysis does not exclude the occurrence of synapses with "presynaptic silent secretion" that cannot be loaded with styryl dyes but can release minute amounts of neurotransmitter to activate NMDA receptors, as reported recently by Renger et al. (2001). However, under the same culture conditions and cell density, the $\mathrm{CA} 1 \rightarrow \mathrm{CA} 3$ cultures used by Renger and colleagues were shown to have a delayed appearance of FM1-43 staining compared with the dentate gyrus $\rightarrow$ CA3-derived cultures used in this study (Kavalali et al., 1999b). In contrast to the CA1 $\rightarrow$ CA3 system, in the current study we detected FM1-43 labeling as early as 5 DIV limiting the prevalence of such synapses.

Time-lapse imaging studies on the sequence of events leading to synapse formation indicate functional vesicle recycling as the earliest indicator of synaptogenesis after the initiation of axodendritic contacts (Ziv and Smith, 1996; Ahmari et al., 2000; Friedman et al., 2000). Some isolated synaptic vesicles in axons also can recycle in an activity-dependent manner before target contact (Matteoli et al., 1992; Dai and Peng, 1996). In our measurements the vesicle clusters that did not respond to hypertonicity presumably belong to the former category because of the presence of a high density of contact sites in these cultures at 5 DIV in addition to the discrete localization and large fluorescence intensity of the puncta, which were not altered significantly during the first $3 \mathrm{~d}$ of the maturation process (Fig. 1B). Mechanistically, the form of vesicle recycling detected in these newborn synapses may be analogous to tetanus toxin-insensitive uptake of antibodies against synaptotagmin lumenal domain detected in isolated axons (Verderio et al., 1999). A detailed understanding of this type of recycling is precluded by our limited knowledge on the kinetic properties of vesicle turnover in synapses with compromised soluble $N$-ethylmaleimide-sensitive factor attachment protein receptor (SNARE) complex constituents (Capogna et al., 1997; Deitcher et al., 1998).

This initial stage is followed by a transitory state during which existing recycling vesicles in the synapse become functionally docked, forming the RRP. Sequential destaining and doublestaining experiments show a wide distribution of RRP to RP ratios with a significantly higher mean compared with ratios found in mature synapses. Synapses that lack synapsin I and II had a lower fraction of vesicles associated with the RRP at this stage, although this reduction in $\mathrm{RRP} / \mathrm{RP}$ ratio was compensated partly with an increase in the recycling pool at mature stages (Fig. 7). However, the size of the recycling pool in mature mutant boutons was distributed more tightly than in wild-type boutons, in agreement with a previous analysis of synapsin I single knock-out (Ryan et al., 1996). Overall, our results suggest a role for synapsins in shaping the characteristics of synapse heterogeneity during maturation by prioritizing the population of RRP. This effect is more pronounced in synapses with small recycling pools.

At the third state of pool organization an increase in the number of recycling vesicles leads to the formation of a reserve pool. At this stage an early scattered distribution of vesicle pool ratios converges to a level in which the number of RRP vesicles is typically between 20 and $30 \%$ of the RP size, and synapses are scaled linearly with respect to their absolute pool sizes with further growth.

Our morphological analysis of spatial distribution of synaptic vesicles in developing boutons is in line with functional results as well as previous quantitative electron microscopic analysis of synapses. A critical observation in developing synapses is the limited growth of synaptic contact zones after initial synaptogenesis, which would confer a physical limitation on the size of individual active zones and maximum available number of functional docking sites therein (Vaughn, 1989). Recent ultrastructural analysis of three dimensionally reconstructed synapses shows large variations in the number of vesicles among boutons in mature stages (Harris and Sultan, 1995; Schikorski and Stevens, 1997). These studies also pointed out a strong correlation between the total number of vesicles in the synapse and the number of morphologically docked vesicles.

\section{Vesicle pool organization as a determinant of presynaptic function}

We can draw direct comparisons between the organizational map of vesicle pools described here and several phenomena observed in synaptic physiology. As stated above, synapses without a RRP 
are functionally impaired and would be presynaptically silent under physiological stimulation. These synapses gradually become functional, presumably after availability and/or maturation of certain active zone components and fusion machinery constituents. Several signal transduction pathways including $\mathrm{Ca}^{2+}$ signaling mechanisms and activation of PKC and PKA potentially can influence this process (Bolshakov et al., 1997; Ma et al., 1999). Our ability to detect these synapses with strong stimulation paradigms supports the presence of such unsilencing mechanisms.

Synapses with a small number of vesicles with a large fraction in a functionally docked state are expected to be severely vulnerable to depression under repetitive stimulation because of the rapid depletion of vesicle supply in the absence of a reserve pool of vesicles. Dynamic changes in functional docking sites under $\mathrm{Ca}^{2+}$-dependent stimulation or by PKC activators may not be a critical factor at this stage because of a shortage of vesicles to support these changes (Smith et al., 1998). However, late development of a reserve pool may provide spare vesicles that could respond to activity-dependent alterations in the number of functional docking sites, thus leading to transient increases in the RRP that would support facilitation. The presence of a RRPspecific recycling scheme as described recently by Pyle et al. (2000) would be critical for synapses that contain solely functionally docked vesicles. To cope with increased demands on their limited vesicle supply, synapses would benefit from a mechanism in which they can reuse the RRP vesicles rapidly.

\section{Multiple degrees of heterogeneity within synapse populations during development}

Currently, we do not know the maturational states of presynaptic terminals in more intact preparations such as brain slices. Interestingly, available data on vesicle recycling in brain slices hint at the presence of synapses with slow stimulation-dependent destaining, with $t_{1 / 2} \sim 200 \mathrm{sec}$ reminiscent of the earliest stage of maturation described here (Pyle et al., 1999). In our experiments the distribution of synaptic vesicle pools described at early stages of maturation was persistent at later stages of development. The hyperbolic decline of pool ratios during synapse maturation indicates relative conservation of the absolute size of the RRP during early synaptic growth. Such a trend would equalize synaptic strengths during low-frequency/short-duration stimulations for a wide range of synapse sizes. Distinctions in reserve pool sizes, however, would diversify responses at sustained high-frequency stimulations. Therefore, our results suggest that the fraction of vesicles in the RRP provides an additional degree of functional heterogeneity among synapses besides the total number of vesicles.

Our experiments pinpoint several possible states presynaptic terminals can be in during maturational time scale. Reversible transitions between modes of pool organization described here may well explain several forms of alterations in presynaptic properties observed during short or more sustained forms of synaptic plasticity.

\section{REFERENCES}

Ahmari SE, Buchanan J, Smith SJ (2000) Assembly of presynaptic active zones from cytoplasmic transport packets. Nat Neurosci 3:445-451.

Amaral DG, Dent JA (1981) Development of the mossy fibers of the dentate gyrus. I. A light and electron microscopic study of the mossy fibers and their expansions. J Comp Neurol 195:51-86.

Baranes D, López-García JC, Chen M, Bailey CH, Kandel ER (1996) Reconstitution of the hippocampal mossy fiber and associational-commissural pathways in a novel dissociated cell culture system. Proc Natl Acad Sci USA 93:4706-4711.

Basarsky TA, Parpura V, Haydon PG (1994) Hippocampal synaptogen- esis in cell culture: developmental time course of synapse formation, calcium influx, and synaptic protein distribution. J Neurosci 14:6402-6411.

Betz WJ, Mao F, Smith CB (1996) Imaging exocytosis and endocytosis. Curr Opin Neurobiol 6:365-371.

Blue ME, Parnavelas JG (1983) The formation and maturation of synapses in the visual cortex of the rat. I. Qualitative analysis. J Neurocytol 12:599-616.

Bolshakov VY, Siegelbaum SA (1995) Regulation of hippocampal transmitter release during development and long-term potentiation. Science 269:1730-1734.

Bolshakov VY, Golan H, Kandel ER, Siegelbaum SA (1997) Recruitment of new sites of synaptic transmission during the cAMP-dependent late phase of LTP at CA3-CA1 synapses in the hippocampus. Neuron 19:635-651.

Capogna M, McKinney RA, O'Connor V, Gahwiler BH, Thompson SM (1997) $\mathrm{Ca}^{2+}$ or $\mathrm{Sr}^{2+}$ partially rescues synaptic transmission in hippocampal cultures treated with botulinum toxin $\mathrm{A}$ and $\mathrm{C}$, but not tetanus toxin. J Neurosci 17:7190-7202.

Dai Z, Peng HB (1996) Dynamics of synaptic vesicles in cultured spinal cord neurons in relationship to synaptogenesis. Mol Cell Neurosci 7:443-452.

Deitcher DL, Ueda A, Stewart BA, Burgess RW, Kidokoro Y, Schwarz TL (1998) Distinct requirements for evoked and spontaneous release of neurotransmitter are revealed by mutations in the Drosophila gene neuronal-synaptobrevin. J Neurosci 18:2028-2039.

Dobrunz LE, Stevens CF (1997) Heterogeneity of release probability, facilitation, and depletion at central synapses. Neuron 18:995-1008.

Durand GM, Kovalchuk Y, Konnerth A (1996) Long-term potentiation and functional synapse induction in developing hippocampus. Nature 381:71-75.

Dyson SE, Jones DG (1980) Quantitation of terminal parameters and their inter-relationships in maturing central synapses: a perspective for experimental studies. Brain Res 183:43-59.

Fernandez-Chacon R, Südhof TC (1999) Genetics of synaptic vesicle function: toward the complete functional anatomy of an organelle. Annu Rev Physiol 61:753-776.

Friedman HV, Bresler T, Garner CC, Ziv NE (2000) Assembly of new individual excitatory synapses: time course and temporal order of synaptic molecule recruitment. Neuron 27:57-69.

Goda Y, Stevens CF (1998) Readily releasable pool size changes associated with long-term depression. Proc Natl Acad Sci USA 95:1283-1288.

Harris KM, Sultan P (1995) Variation in the number, location, and size of synaptic vesicles provides an anatomical basis for the nonuniform probability of release at hippocampal CA1 synapses. Neuropharmacology 34:1387-1395.

Hessler NA, Shirke AM, Malinow R (1993) The probability of transmitter release at a mammalian central synapse. Nature 366:569-572.

Hilfiker S, Pieribone VA, Czernik AJ, Kao HT, Augustine GJ, Greengard P (1999) Synapsins as regulators of neurotransmitter release. Philos Trans R Soc Lond B Biol Sci 354:269-279.

Hsia AY, Malenka RC, Nicoll RA (1998) Development of excitatory circuitry in the hippocampus. J Neurophysiol 79:2013-2024.

Kavalali ET, Klingauf J, Tsien RW (1999a) Properties of fast endocytosis at hippocampal synapses. Philos Trans R Soc Lond B Biol Sci 354:337-346.

Kavalali ET, Klingauf J, Tsien RW (1999b) Activity-dependent regulation of synaptic clustering in a hippocampal culture system. Proc Natl Acad Sci USA 96:12893-12900.

Klingauf J, Kavalali ET, Tsien RW (1998) Kinetics and regulation of fast endocytosis at hippocampal synapses. Nature 394:581-585.

Li L, Chin LS, Shupliakov O, Brodin L, Sihra TS, Hvalby O, Jensen V, Zheng D, McNamara JO, Greengard P, Andersen P (1995) Impairment of synaptic vesicle clustering and of synaptic transmission, and increased seizure propensity, in synapsin I-deficient mice. Proc Natl Acad Sci USA 92:9235-9239.

Ma L, Zablow L, Kandel ER, Siegelbaum SA (1999) Cyclic AMP induces functional presynaptic boutons in hippocampal CA3-CA1 neuronal cultures. Nat Neurosci 2:24-30.

Maccaferri G, Toth K, McBain CJ (1998) Target-specific expression of presynaptic mossy fiber plasticity. Science 279:1368-1370.

Markram H, Wang Y, Tsodyks M (1998) Differential signaling via the same axon of neocortical pyramidal neurons. Proc Natl Acad Sci USA 95:5323-5328.

Matteoli M, Takei K, Perin MS, Südhof TC, De Camilli P (1992) Exoendocytotic recycling of synaptic vesicles in developing processes of cultured hippocampal neurons. J Cell Biol 117:849-861.

Murthy VN, Stevens CF (1999) Reversal of synaptic vesicle docking at central synapses. Nat Neurosci 2:503-507.

Murthy VN, Sejnowski TJ, Stevens CF (1997) Heterogeneous release properties of visualized individual hippocampal synapses. Neuron 18:599-612.

Neher E, Zucker RS (1993) Multiple calcium-dependent processes related to secretion in bovine chromaffin cells. Neuron 10:21-30. 
Neves G, Lagnado L (1999) The kinetics of exocytosis and endocytosis in the synaptic terminal of goldfish retinal bipolar cells. J Physiol (Lond) 515:181-202.

Pieribone VA, Shupliakov O, Brodin L, Hilfiker-Rothenfluh S, Czernik AJ, Greengard P (1995) Distinct pools of synaptic vesicles in neurotransmitter release. Nature 375:493-497.

Pouzat C, Hestrin S (1997) Developmental regulation of basket/stellate cell $\rightarrow$ Purkinje cell synapses in the cerebellum. J Neurosci 17:9104-9112.

Pyle JL, Kavalali ET, Choi S, Tsien RW (1999) Visualization of synaptic activity in hippocampal slices with FM1-43 enabled by fluorescence quenching. Neuron 24:803-808.

Pyle JL, Kavalali ET, Piedras-Renteria ES, Tsien RW (2000) Rapid reuse of readily releasable pool vesicles at hippocampal synapses. Neuron 28:221-231.

Renger JJ, Egles C, Liu G (2001) A developmental switch in neurotransmitter flux enhances synaptic efficacy by affecting AMPA receptor activation. Neuron 29:469-484.

Reyes A, Sakmann B (1999) Developmental switch in the short-term modification of unitary EPSPs evoked in layer $2 / 3$ and layer 5 pyramidal neurons of rat neocortex. J Neurosci 19:3827-3835.

Reyes A, Lujan R, Rozov A, Burnashev N, Somogyi P, Sakmann B (1998) Target cell-specific facilitation and depression in neocortical circuits. Nat Neurosci 1:279-285.

Rosahl TW, Geppert M, Spillane D, Herz J, Hammer RE, Malenka RC, Südhof TC (1993) Short-term synaptic plasticity is altered in mice lacking synapsin I. Cell 75:661-670.

Rosahl TW, Spillane D, Missler M, Herz J, Selig DK, Wolff JR, Hammer RE, Malenka RC, Südhof TC (1995) Essential functions of synapsins I and II in synaptic vesicle regulation. Nature 375:488-493.

Rosenmund C, Stevens CF (1996) Definition of the readily releasable pool of vesicles at hippocampal synapses. Neuron 16:1197-1207.

Rosenmund C, Clements JD, Westbrook GL (1993) Non-uniform probability of glutamate release at a hippocampal synapse. Science $262: 754-757$
Ryan TA, Smith SJ (1995) Vesicle pool mobilization during action potential firing at hippocampal synapses. Neuron 14:983-989.

Ryan TA, Reuter H, Wendland B, Schweizer FE, Tsien RW, Smith SJ (1993) The kinetics of synaptic vesicle recycling measured at single presynaptic boutons. Neuron 11:713-724.

Ryan TA, Li L, Chin LS, Greengard P, Smith SJ (1996) Synaptic vesicle recycling in synapsin I knock-out mice. J Cell Biol 134:1219-1227.

Schikorski T, Stevens CF (1997) Quantitative ultrastructural analysis of hippocampal excitatory synapses. J Neurosci 17:5858-5867.

Schneggenburger R, Meyer AC, Neher E (1999) Released fraction and total size of a pool of immediately available transmitter quanta at a calyx synapse. Neuron 23:399-409.

Smith C, Moser T, Xu T, Neher E (1998) Cytosolic $\mathrm{Ca}^{2+}$ acts by two separate pathways to modulate the supply of release-competent vesicles in chromaffin cells. Neuron 20:1243-1253.

Vaughn JE (1989) Fine structure of synaptogenesis in the vertebrate central nervous system. Synapse 3:255-285.

Verderio C, Coco S, Bacci A, Rossetto O, De Camilli P, Montecucco C, Matteoli M (1999) Tetanus toxin blocks the exocytosis of synaptic vesicles clustered at synapses, but not of synaptic vesicles in isolated axons. J Neurosci 19:6723-6732.

von Gersdorff H, Schneggenburger R, Weis S, Neher E (1997) Presynaptic depression at a calyx synapse: the small contribution of metabotropic glutamate receptors. J Neurosci 17:8137-8146.

Wu G, Malinow R, Cline HT (1996) Maturation of a central glutamatergic synapse. Science 274:972-976.

Wu LG, Betz WJ (1998) Kinetics of synaptic depression and vesicle recycling after tetanic stimulation of frog motor nerve terminals. Biophys J 74:3003-3009.

Wu LG, Borst JG (1999) The reduced release probability of releasable vesicles during recovery from short-term synaptic depression. Neuron 23:821-832

Ziv NE, Smith SJ (1996) Evidence for a role of dendritic filopodia in synaptogenesis and spine formation. Neuron 17:91-102.

Zucker RS (1989) Short-term synaptic plasticity. Annu Rev Neurosci 12:13-31. 